\title{
Author Correction: Emerging photoluminescence from the dark-exciton phonon replica in monolayer $\mathrm{WSe}_{2}$
}

\author{
Zhipeng Li (10) 1,2,13, Tianmeng Wang 1,13, Chenhao Jin (10 3,13, Zhengguang Lu 4,5, Zhen Lian', Yuze Meng ${ }^{1,6}$, \\ Mark Blei ${ }^{7}$, Shiyuan Gao ${ }^{8}$, Takashi Taniguchi ${ }^{9}$, Kenji Watanabe ${ }^{9}{ }^{9}$, Tianhui Ren ${ }^{2 \star}$, Sefaattin Tongay ${ }^{7}$, Li Yang ${ }^{8}$, \\ Dmitry Smirnov (iD ${ }^{4}$, Ting Cao ${ }^{10,11 \star} \&$ Su-Fei Shi (iD) ${ }^{1,12 \star}$
}

Correction to: Nature Communications https://doi.org/10.1038/s41467-019-10477-6, published online 06 June 2019.

The original version of this Article omitted the following from the end of the Acknowledgements: 'Z.Lu and D.S. acknowledge support from the US Department of Energy (DE-FG02-07ER46451) for magneto-optical measurements performed at the National High Magnetic Field Laboratory, which is supported by the National Science Foundation Cooperative Agreement No. DMR-1644779 and the State of Florida'. This has now been corrected in both the PDF and HTML versions of the Article.

Published online: 11 October 2019

Open Access This article is licensed under a Creative Commons Attribution 4.0 International License, which permits use, sharing, adaptation, distribution and reproduction in any medium or format, as long as you give appropriate credit to the original author(s) and the source, provide a link to the Creative Commons license, and indicate if changes were made. The images or other third party material in this article are included in the article's Creative Commons license, unless indicated otherwise in a credit line to the material. If material is not included in the article's Creative Commons license and your intended use is not permitted by statutory regulation or exceeds the permitted use, you will need to obtain permission directly from the copyright holder. To view a copy of this license, visit http://creativecommons.org/licenses/by/4.0/.
\end{abstract}

(c) The Author(s) 2019

\footnotetext{
${ }^{1}$ Department of Chemical and Biological Engineering, Rensselaer Polytechnic Institute, Troy, NY 12180, USA. ${ }^{2}$ School of Chemistry and Chemical Engineering, Shanghai Jiao Tong University, 200240 Shanghai, China. ${ }^{3}$ Kavli Institute at Cornell for Nanoscale Science, Ithaca, NY 14853, USA. ${ }^{4}$ National High Magnetic Field Lab, Tallahassee, FL 32310, USA. ${ }^{5}$ Department of Physics, Florida State University, Tallahassee, FL 32306, USA. ${ }^{6}$ College of Physics, Nanjing University, 210093 Nanjing, China. ${ }^{7}$ School for Engineering of Matter, Transport and Energy, Arizona State University, Tempe, AZ 85287, USA. ${ }^{8}$ Department of Physics, Washington University in St. Louis, St. Louis, MO 63136, USA. ${ }^{9}$ National Institute for Materials Science, 1-1 Namiki, Tsukuba 305-0044, Japan. ${ }^{10}$ Geballe Laboratory for Advanced Materials, Stanford University, Stanford, CA 94305, USA. ${ }^{11}$ Department of Materials Science and Engineering, University of Washington, Seattle, WA 98195, USA. ${ }^{12}$ Department of Electrical, Computer \& Systems Engineering, Rensselaer Polytechnic Institute, Troy, NY 12180, USA.

${ }^{13}$ These authors contributed equally: Zhipeng Li, Tianmeng Wang, Chenhao Jin. *email: thren@sjtu.edu.cn; tingcao@uw.edu; shis2@rpi.edu
} 